\title{
Lopsidedness in WHISP galaxies
}

\section{Rotation curves and kinematic lopsidedness $\star$}

\author{
J. van Eymeren ${ }^{1}$, E. Jütte ${ }^{2}$, C. J. Jog ${ }^{3}$, Y. Stein ${ }^{2}$, and R.-J. Dettmar ${ }^{2}$ \\ ${ }^{1}$ Fakultät für Physik, Universität Duisburg-Essen, Lotharstr. 1, 47048 Duisburg, Germany \\ e-mail: janine.vaneymeren@uni-due.de \\ 2 Astronomisches Institut der Ruhr-Universität Bochum, Universitätsstr. 150, 44780 Bochum, Germany \\ e-mail: eva.juette@astro.rub.de \\ 3 Department of Physics, Indian Institute of Science, 560012 Bangalore, India \\ e-mail: cjjog@physics.iisc.ernet.in
}

Received 22 November 2010 / Accepted 19 March 2011

\begin{abstract}
The frequently observed lopsidedness of the distribution of stars and gas in disc galaxies is still considered as a major problem in galaxy dynamics. It is even discussed as an imprint of the formation history of discs and the evolution of baryons in dark matter haloes. Here, we analyse a selected sample of 70 galaxies from the Westerbork Hi Survey of Spiral and Irregular Galaxies. The Hi data allow us to follow the morphology and the kinematics out to very large radii. In the present paper, we present the rotation curves and study the kinematic asymmetry. We extract the rotation curves of the receding and approaching sides separately and show that the kinematic behaviour of disc galaxies can be classified into five different types: symmetric velocity fields where the rotation curves of the receding and approaching sides are almost identical; global distortions where the rotation velocities of the receding and approaching sides have an offset that is constant with radius; local distortions leading to large deviations in the inner and negligible deviations in the outer parts (and vice versa); and distortions that divide the galaxies into two kinematic systems that are visible in terms of the different behaviour of the rotation curves of the receding and approaching sides, which leads to a crossing and a change in side. The kinematic lopsidedness is measured from the maximum rotation velocities, averaged over the plateau of the rotation curves. This gives a good estimate of the global lopsidedness in the outer parts of the sample galaxies. We find that the mean value of the perturbation parameter denoting the lopsided potential as obtained from the kinematic data is 0.056 . Altogether, $36 \%$ of the sample galaxies are globally lopsided, which can be interpreted as the disc responding to a halo that was distorted by a tidal encounter. In Paper II, we study the morphological lopsidedness of the same sample of galaxies.
\end{abstract}

Key words. surveys - galaxies: evolution - galaxies: ISM - galaxies: kinematics and dynamics - galaxies: structure

\section{Introduction}

It has been known for many years that the discs of galaxies often display a large-scale asymmetry in their morphology, prominent examples being M 101, NGC 891, and NGC 4565. This asymmetry was described for the first time by Baldwin et al. (1980), who defined the term "lopsidedness". The first systematic study was performed by Rix \& Zaritsky (1995), who investigated near-infrared images of a sample of disc galaxies and characterised the lopsidedness of the stellar distribution in terms of the $m=1$ mode of a Fourier analysis. They found that at least $30 \%$ of the stellar discs of galaxies are significantly lopsided. A comparable study of gaseous discs was difficult to perform because spatially resolved maps of a large sample were then unavailable. Therefore, Richter \& Sancisi (1994) investigated a large number of integrated $\mathrm{H}_{\mathrm{I}}$ spectra and found that up to $50 \%$ display an asymmetric global Hi profile that was assumed to be caused by lopsidedness. The advantage of this method is that it can be readily applied to analyse a large dataset. However, it is unclear whether an asymmetric H i profile is caused by either an asymmetric gas distribution or kinematic lopsidedness. The first twodimensional Fourier decomposition of $\mathrm{H}$ I surface density maps,

\footnotetext{
* Appendix $\mathrm{A}$ is available in electronic form at http://www. aanda.org
}

analogous to the stellar studies (e.g., by Rix \& Zaritsky 1995), was done by Angiras et al. (2006, 2007), who showed that the HI gas distribution in group galaxies is significantly lopsided. For more details about lopsided galaxies, we refer to the review by Jog \& Combes (2009).

Lopsidedness is observed not only in the morphology of galaxies, but also in the kinematics. Rotation curves are usually derived to obtain the mass distribution in a galaxy that is rotationally supported (e.g., Binney \& Tremaine 1987). The typical assumption is that the rotation curves are azimuthally symmetric. However, observations detect local deviations from a smooth circular rotation of a few $\mathrm{km} \mathrm{s}^{-1}$, which are contributed to streaming motions caused by either spiral arms (Shane \& Bieger-Smith 1966) or bars (Rhee et al. 2004). In addition, Huchtmeier (1975) showed that the rotation curves derived from the two halves of a galactic disc are also asymmetric on large scales. The difference in the rotation velocities was found to be $\geq 20 \mathrm{~km} \mathrm{~s}^{-1}$. Most recent studies reveal that large-scale kinematic asymmetries are a common phenomenon, as shown by both H I studies (e.g., Kannappan \& Fabricant 2001; Swaters et al. 2009) and $\mathrm{H} \alpha$ studies (e.g., the GHASP work by Epinat et al. 2008).

The origin of the asymmetry in the rotation curves was also addressed theoretically by Jog (2002), who showed that the global asymmetry in the rotation curves as well as in the 
morphology can be caused by the stars and gas in a galactic disc responding to a distorted halo potential. Even a small lopsided perturbation potential results in highly disturbed kinematics (Jog 1997, 2002), which should therefore be easy to detect.

An estimate of the perturbation in a lopsided potential that gives rise to the kinematic lopsidedness can be retrieved from the rotation curves of the receding and approaching sides. Therefore, it is crucial to accurately define the kinematic parameters, i.e., the systemic velocity, the coordinates of the dynamic centre, the inclination, and the position angle. As the perturbation parameter for the lopsided potential is calculated from the maximum rotation velocities, sensitive data that trace the gas to large radii, where the rotation curve ends in a plateau, are needed. Interferometric $\mathrm{H}_{\mathrm{I}}$ observations are the most suitable for this kind of study.

In this first of two papers, we introduce the sample, derive rotation curves by performing a tilted-ring analysis, and try to quantify the kinematic lopsidedness. The morphological lopsidedness is analysed in a second, companion paper (van Eymeren et al. 2011, Paper II from now on).

This paper is organised as follows. In Sect. 2, we outline the data selection process, and in Sect. 3 we describe the data analysis. In Sect. 4, we present the results, which are discussed in Sect. 5. We end the paper with a brief summary in Sect. 6.

\section{Sample selection}

This paper is based on data from the Westerbork H I Survey of Irregular and SPiral galaxies (WHISP ${ }^{1}$ ). A detailed description of the survey and data reduction process is given in Swaters et al. (2002). Three cubes of different spatial resolution per galaxy were produced by the WHISP team, a full resolution cube, one cube smoothed to $30^{\prime \prime} \times 30^{\prime \prime}$, and one cube smoothed to $60^{\prime \prime} \times 60^{\prime \prime}$. To combine reasonably high spatial resolution with a sufficiently high signal-to-noise ratio $(\mathrm{S} / \mathrm{N})$, the following analysis is based on the $30^{\prime \prime} \times 30^{\prime \prime}$ data.

We included only galaxies with inclinations ranging between $20^{\circ}$ and $75^{\circ}$. This range was chosen because the velocity information extracted for a nearly face-on galaxy is less than that for one that is edge-on, which results in a larger uncertainty in measuring the inclination. However, reliable velocity information can be obtained for nearly edge-one galaxy, but the surface density map will not be suitable for the analysis of the morphological lopsidedness. Therefore, the above-mentioned range of inclinations appears to be a good compromise.

To be able to measure lopsidedness to large radii, we next limited the sample to galaxies with a ratio of $\mathrm{H}_{\mathrm{I}}$ diameter to beam size larger than 10 , using the $30^{\prime \prime} \times 30^{\prime \prime}$ data. From the resulting sample, we had to eliminate about 30 galaxies either because the $\mathrm{S} / \mathrm{N}$ was low causing the $\mathrm{H}_{\mathrm{I}}$ intensity distribution to be very patchy or because the velocity field was too distorted to allow an analysis on global scales. This also excluded galaxies with pronounced warps, visible in the morphology of the nearly edge-on cases or in the velocity field of galaxies of low inclination. This left us with 70 galaxies. Some general properties of the final sample galaxies are given in Table 1.

Making sure that these selection criteria do not bias our sample towards bright objects and only certain morphological types, we considered the range of absolute $B$-magnitudes and morphological types. We found that the galaxies are evenly distributed from -23 to $-15 B$-magnitudes (Fig. 1, upper panel). We cover

\footnotetext{
${ }^{1}$ http://www.astro.rug.nl/ whisp/
}
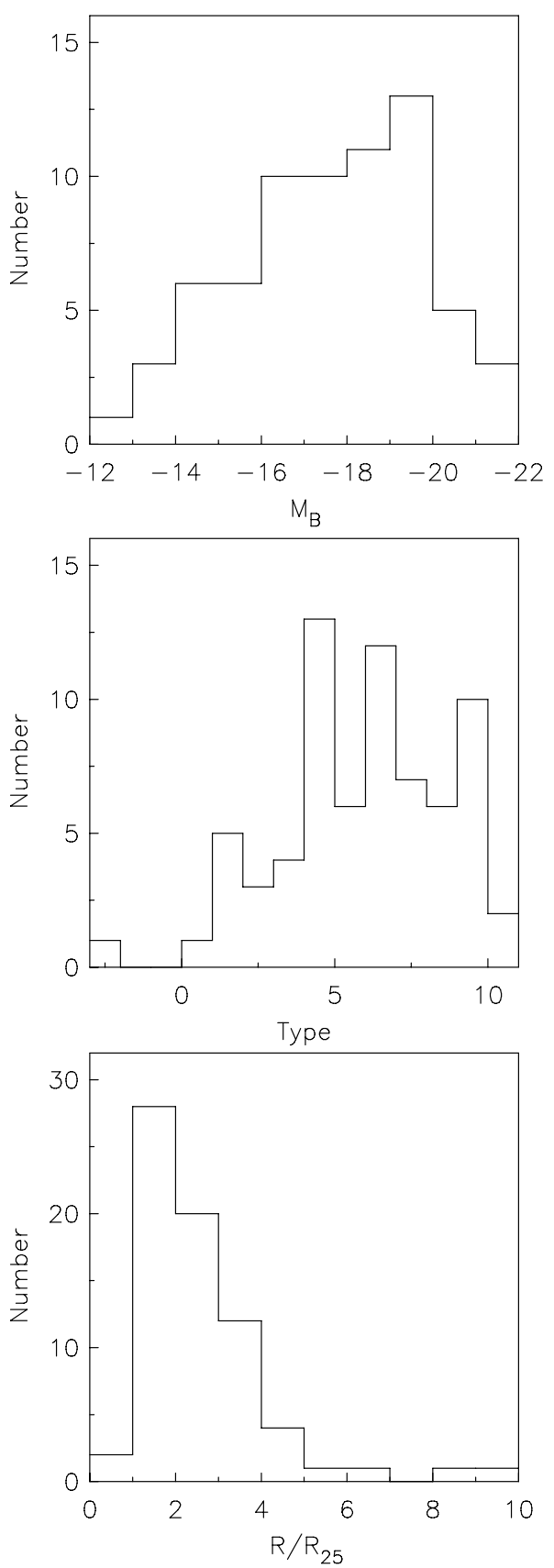

Fig. 1. The distribution of the selected galaxies over absolute $B$ magnitude (upper panel), morphological type (middle panel), and radial extent (lower panel).

a range of morphological types including a few early-type spirals and an increasing number density towards late-type spiral and irregular galaxies (Fig. 1, middle panel). Most galaxies display emission, which is extended from 1 to $4 R_{25}$ (Fig. 1 , lower panel). However, our sample also covers a few galaxies whose H I emission could be detected to 6 to $10 R_{25}\left(R_{25}\right.$ being the apparent optical radius).

\section{Data analysis}

The data analysis is based on routines within the Groningen Image Processing System (GIPSY ${ }^{2}$, van der Hulst et al. 1992).

2 http://www.astro.rug.nl/ gipsy/ 
Table 1. General parameters of all sample galaxies.

\begin{tabular}{|c|c|c|c|c|c|c|c|c|}
\hline $\begin{array}{l}\text { Galaxy } \\
\text { (1) } \\
\end{array}$ & $\begin{array}{c}\text { Other name } \\
\text { (2) }\end{array}$ & $\begin{array}{c}\text { Hubble type } \\
\text { (3) }\end{array}$ & $\begin{array}{c}\alpha(\mathrm{J} 2000.0) \\
{[\mathrm{h} \mathrm{m} \mathrm{s}]} \\
(4)\end{array}$ & $\begin{array}{c}\delta(\mathrm{J} 2000.0) \\
{\left[\begin{array}{c}{ }^{\circ} \prime \\
\prime \prime\end{array}\right]} \\
(5) \\
\end{array}$ & $\begin{array}{c}M_{B} \\
{[\mathrm{mag}]} \\
(6) \\
\end{array}$ & $\begin{array}{c}D \\
{[\mathrm{Mpc}]} \\
(7) \\
\end{array}$ & $\begin{array}{c}R_{25} \\
{[\mathrm{kpc}]} \\
(8) \\
\end{array}$ & $\begin{array}{c}R_{\max } \\
{\left[R / R_{25}\right]} \\
(9) \\
\end{array}$ \\
\hline UGC 625 & IC 65 & 4 & 010055.4 & +474055.1 & -19.20 & $37.3^{\mathrm{H}}$ & 13.94 & 2.33 \\
\hline UGC 731 & $\ldots$ & 9.9 & $\begin{array}{lll}01 & 10 & 44.0\end{array}$ & $\begin{array}{r}+493607.9 \\
\end{array}$ & -12.74 & $8.0^{\mathrm{S}}$ & 2.17 & 3.49 \\
\hline UGC 1249 & IC 1727 & 8.9 & 014729.9 & +272000.1 & -16.33 & $7.5^{\mathrm{s}}$ & 7.05 & 1.01 \\
\hline UGC 1256 & NGC 672 & 6 & 014754.5 & $\begin{array}{r}272558.0 \\
\end{array}$ & -17.37 & $7.2^{\mathrm{E}}$ & 7.42 & 1.20 \\
\hline UGC 1281 & $\ldots$ & 7.5 & 014932.0 & +323523.0 & -13.81 & $5.5^{\mathrm{S}}$ & 4.10 & 1.27 \\
\hline UGC 1317 & NGC 674 & 4.9 & 015117.6 & +222128.7 & -20.34 & $42.2^{\mathrm{E}}$ & 23.33 & 1.84 \\
\hline UGC 1501 & NGC 784 & 7.8 & 020116.9 & +285014.1 & -14.97 & $5.7^{\mathrm{H}}$ & 3.46 & 1.80 \\
\hline UGC 1913 & NGC 925 & 7 & 022716.9 & +333445.0 & -18.74 & $9.3^{\mathrm{E}}$ & 14.52 & 1.07 \\
\hline UGC 2034 & 2 & 9.8 & 023343.0 & +403141.2 & -15.82 & $10.1^{\mathrm{S}}$ & 4.43 & 1.99 \\
\hline UGC 2080 & IC 239 & 6 & 023627.9 & +385811.7 & -18.69 & $13.7^{\mathrm{E}}$ & 8.50 & 2.70 \\
\hline UGC 2455 & NGC 1156 & 9.9 & 025942.2 & +251414.2 & -16.26 & $7.8^{\mathrm{S}}$ & 3.27 & 2.08 \\
\hline UGC 2800 & $\ldots$ & 10 & 034002.5 & $\begin{array}{r}+712421.1 \\
\end{array}$ & -14.57 & $20.6^{\mathrm{E}}$ & 7.02 & 2.56 \\
\hline UGC 2855 & $\cdots$ & 5 & 034820.7 & +700758.4 & -17.61 & $17.5^{\mathrm{E}}$ & 9.03 & 1.55 \\
\hline UGC 2953 & IC 356 & 3.4 & 040746.9 & $\begin{array}{r}+694844.8 \\
\end{array}$ & -19.08 & $15.1^{\mathrm{N}}$ & 8.74 & 2.39 \\
\hline UGC 3273 & $\ldots$ & 9 & 051744.4 & +533304.6 & -14.86 & $12.2^{\mathrm{E}}$ & 4.56 & 2.92 \\
\hline UGC 3371 & $\cdots$ & 9.9 & 055638.6 & $\begin{array}{r}+751858.0 \\
\end{array}$ & -15.57 & $12.8^{\mathrm{S}}$ & 7.08 & 1.45 \\
\hline UGC 3574 & $\cdots$ & 5.8 & 065310.4 & +571040.0 & -18.31 & $21.8^{\mathrm{E}}$ & 4.69 & 3.72 \\
\hline UGC 3580 & $\begin{array}{l}\cdots \\
\ldots\end{array}$ & 1.1 & 065530.9 & +693347.0 & -18.40 & $19.2^{\mathrm{N}}$ & 5.97 & 3.27 \\
\hline UGC 3734 & NGC 2344 & 4.4 & 071228.7 & +471000.1 & -17.85 & $15.9^{\mathrm{E}}$ & 4.83 & 2.63 \\
\hline UGC 3851 & NGC 2366 & 9.8 & 072854.7 & $\begin{array}{r}+691256.8 \\
\end{array}$ & -13.80 & $3.4^{\mathrm{S}}$ & 2.16 & 2.86 \\
\hline UGC 4173 & & 9.9 & 080712.1 & +800730.0 & -15.09 & $16.8^{S}$ & 1.54 & 9.50 \\
\hline UGC 4278 & IC 2233 & 6.4 & 081358.9 & $\begin{array}{r}+454431.7 \\
\end{array}$ & -16.31 & $10.5^{\mathrm{S}}$ & 4.40 & 2.08 \\
\hline UGC 4284 & NGC 2541 & 6 & 081440.1 & $\begin{array}{r}+490342.2 \\
\end{array}$ & -17.15 & $9.8^{\mathrm{E}}$ & 4.30 & 3.48 \\
\hline UGC 4458 & NGC 2599 & 1 & 083211.3 & $\begin{array}{r}\text { + } 223338.0 \\
\end{array}$ & -21.01 & $64.2^{\mathrm{N}}$ & 14.46 & 3.55 \\
\hline UGC 4543 & & 7.9 & 084321.6 & $\begin{array}{r}+454408.4 \\
\end{array}$ & -17.87 & $30.3^{\mathrm{S}}$ & 5.55 & 4.37 \\
\hline UGC 4838 & NGC 2776 & 5.2 & 091214.5 & $\begin{array}{r}\text { T } 5717.4 \\
\end{array}$ & -20.67 & $37.3^{\mathrm{H}}$ & 11.59 & 2.57 \\
\hline UGC 5079 & NGC 2903 & 4 & 093210.1 & $\begin{array}{r}-213003.0 \\
\end{array}$ & -19.76 & $8.9^{\mathrm{H}}$ & 15.25 & 1.40 \\
\hline UGC 5251 & NGC 3003 & 4.3 & 094836.1 & $\begin{array}{r}+332517.4 \\
\end{array}$ & -19.23 & $21.5^{\mathrm{E}}$ & 14.97 & 1.36 \\
\hline UGC 5253 & NGC 2985 & 2.4 & 095022.2 & +721643.1 & -20.14 & $21.1^{\mathrm{E}}$ & 11.14 & 1.65 \\
\hline UGC 5532 & NGC 3147 & 3.9 & 101653.7 & $\begin{array}{r}+732402.7 \\
\end{array}$ & -21.53 & $41.1^{\mathrm{E}}$ & 24.35 & 1.35 \\
\hline UGC 5685 & NGC 3254 & 4 & 102919.9 & +292930.6 & -18.96 & $19.9^{\mathrm{H}}$ & 6.78 & 2.77 \\
\hline UGC 5717 & NGC 3259 & 3.7 & 103234.9 & $\begin{array}{r}+650227.9 \\
+65\end{array}$ & ... & $25.7^{\mathrm{H}}$ & 6.49 & 4.60 \\
\hline UGC 5721 & NGC 3274 & 6.6 & 103217.3 & +274007.6 & -16.15 & $6.7^{\mathrm{S}}$ & 1.58 & 4.62 \\
\hline UGC 5789 & NGC 3319 & 6 & 103909.5 & +414112.0 & -18.58 & $14.1^{\mathrm{E}}$ & 7.44 & 2.20 \\
\hline UGC 5829 & $\ldots$ & 9.8 & 104241.9 & +342656.0 & -15.98 & $9.0^{\mathrm{S}}$ & 5.85 & 1.46 \\
\hline UGC 5918 & $\begin{array}{l}\cdots \\
\ldots\end{array}$ & 10 & 104936.5 & $\begin{array}{r}+653150.0 \\
+\end{array}$ & -13.27 & $7.7^{\mathrm{S}}$ & 2.81 & 2.19 \\
\hline UGC 5997 & NGC 3403 & 4 & 105354.9 & +734125.3 & -18.25 & $20.4^{\mathrm{H}}$ & 8.17 & 2.54 \\
\hline UGC 6225 & NGC 3556 & 6 & 111131.0 & +554026.8 & -19.25 & $12.4^{\mathrm{H}}$ & 7.18 & 2.26 \\
\hline UGC 6446 & $\ldots$ & 6.6 & 112640.5 & +534448.0 & -16.22 & $12.0^{\mathrm{S}}$ & 2.46 & 3.89 \\
\hline UGC 6537 & NGC 3726 & 5.1 & 113321.1 & +470145.1 & -19.49 & $14.3^{\mathrm{E}}$ & 10.92 & 1.33 \\
\hline UGC 6787 & NGC 3898 & 1.7 & 114915.4 & +560503.7 & -19.47 & $18.9^{\mathrm{E}}$ & 9.53 & 2.88 \\
\hline UGC 6937 & NGC 3992 & 4 & 115736.0 & +532228.3 & -20.22 & $17.1^{\mathrm{H}}$ & 20.24 & 0.86 \\
\hline UGC 7081 & NGC 4088 & 4.7 & 120534.2 & +503220.5 & -18.96 & $13.2^{\mathrm{H}}$ & 13.60 & 0.99 \\
\hline UGC 7090 & NGC 4095 & 5.3 & 120601.1 & +472842.4 & -18.00 & $10.6^{\mathrm{H}}$ & 8.67 & 1.07 \\
\hline UGC 7095 & NGC 4100 & 4.1 & 120608.5 & +493457.7 & -18.98 & $17.4^{\mathrm{H}}$ & 11.57 & 1.42 \\
\hline UGC 7151 & NGC 4144 & 6 & 120958.6 & $\begin{array}{r}462725.8 \\
\end{array}$ & -15.78 & $3.5^{\mathrm{s}}$ & 2.67 & 1.14 \\
\hline UGC 7256 & NGC 4203 & -2.7 & 121505.1 & $\begin{array}{r}+31150.4 \\
+\end{array}$ & -19.09 & $16.9^{\mathrm{N}}$ & 8.33 & 2.66 \\
\hline UGC 7321 & $\ldots$ & 6.6 & 121734.0 & +223224.5 & -14.62 & $7.2^{\mathrm{H}}$ & 5.01 & 1.25 \\
\hline UGC 7323 & NGC 4242 & 7.9 & 121730.2 & $\begin{array}{r}+453709.5 \\
\end{array}$ & -17.86 & $8.1^{\mathrm{S}}$ & 4.48 & 1.58 \\
\hline UGC 7353 & NGC 4258 & 4 & 121857.5 & $\begin{array}{r}+471814.3 \\
\end{array}$ & -19.87 & $7.8^{\mathrm{H}}$ & 19.46 & 1.27 \\
\hline UGC 7524 & NGC 4395 & $\begin{array}{c}4 \\
8.9\end{array}$ & 122548.9 & $\begin{array}{r}+41014.5 \\
+333248.7\end{array}$ & $\begin{array}{l}-19.01 \\
-17.59\end{array}$ & $\begin{array}{l}1.0 \\
3.5^{\mathrm{S}}\end{array}$ & $\begin{array}{l}19.40 \\
2.12\end{array}$ & $\begin{array}{l}1.21 \\
3.84\end{array}$ \\
\hline UGC 7603 & NGC 4455 & 7 & 122844.1 & +224913.6 & -16.80 & $6.8^{\mathrm{S}}$ & 1.60 & 4.32 \\
\hline UGC 7766 & NGC 4559 & 6 & 123557.7 & $\begin{array}{r}+275735.1 \\
\end{array}$ & -19.81 & $13.0^{\mathrm{E}}$ & 19.84 & 1.58 \\
\hline UGC 7989 & NGC 4725 & 2.2 & 125026.6 & $\begin{array}{r}+253002.7 \\
\end{array}$ & -21.01 & $18.2^{\mathrm{N}}$ & 25.91 & 1.07 \\
\hline UGC 8863 & NGC 5377 & 1 & 135616.7 & $\begin{array}{r}+471408.5 \\
\end{array}$ & -19.74 & $27.2^{\mathrm{E}}$ & 14.36 & 1.65 \\
\hline UGC 9133 & NGC 5533 & 2.4 & 141607.7 & $\begin{array}{r}+352037.8 \\
\end{array}$ & -20.95 & $54.3^{\mathrm{N}}$ & 22.77 & 3.47 \\
\hline UGC 9211 & $\ldots$ & 9.9 & 142232.2 & $\begin{array}{r}+452301.9 \\
\end{array}$ & -14.89 & $12.6^{S}$ & 1.16 & 8.72 \\
\hline UGC 9649 & NGC 5832 & 3 & 145745.7 & $\begin{array}{r}+714056.4 \\
\end{array}$ & -16.06 & $7.7^{\mathrm{E}}$ & 2.08 & 3.76 \\
\hline UGC 9858 & $\ldots$ & 4 & 152641.5 & $\begin{array}{r}+403352.2 \\
\end{array}$ & ... & $38.2^{\mathrm{E}}$ & 21.61 & 1.29 \\
\hline UGC 10359 & NGC 6140 & 5.6 & 162058.2 & +652326.0 & $\begin{array}{c}\ldots \\
-18.70\end{array}$ & $16.0^{\mathrm{E}}$ & 4.86 & 3.83 \\
\hline UGC 10470 & NGC 6217 & 4 & 163239.2 & $\begin{array}{r}+781153.4 \\
\end{array}$ & -19.59 & $21.2^{\mathrm{E}}$ & 6.90 & 3.35 \\
\hline
\end{tabular}


Table 1. continued.

\begin{tabular}{|c|c|c|c|c|c|c|c|c|}
\hline Galaxy & $\begin{array}{c}\text { Other name } \\
\text { (2) }\end{array}$ & $\begin{array}{c}\text { Hubble type } \\
\text { (3) }\end{array}$ & $\begin{array}{c}\alpha(\mathrm{J} 2000.0) \\
{[\mathrm{h} \mathrm{m} \mathrm{s}]} \\
(4)\end{array}$ & $\begin{array}{c}\delta(\mathrm{J} 2000.0) \\
{\left[\begin{array}{c}{ }^{\circ}{ }^{\prime} \\
\prime \prime\end{array}\right]} \\
(5)\end{array}$ & $\begin{array}{c}M_{B} \\
{[\mathrm{mag}]} \\
(6)\end{array}$ & $\begin{array}{c}D \\
{[\mathrm{Mpc}]} \\
(7)\end{array}$ & $\begin{array}{c}R_{25} \\
{[\mathrm{kpc}]} \\
(8) \\
\end{array}$ & $\begin{array}{c}R_{\max } \\
{\left[R / R_{25}\right]} \\
(9) \\
\end{array}$ \\
\hline UGC 11670 & NGC 7013 & 0.5 & 210333.6 & +295350.6 & -17.77 & $12.7^{\mathrm{E}}$ & 7.70 & 1.56 \\
\hline UGC 11707 & $\ldots$ & 8 & 211431.8 & +264404.5 & -16.00 & $15.9^{\mathrm{S}}$ & 2.37 & 6.35 \\
\hline UGC 11852 & $\ldots$ & 1 & 215559.3 & +275354.3 & -19.83 & $80.0^{\mathrm{E}}$ & 10.61 & 5.48 \\
\hline UGC 11861 & $\ldots$ & 7.8 & 215619.4 & +731513.6 & -17.37 & $25.1^{\mathrm{S}}$ & 6.49 & 3.09 \\
\hline UGC 11891 & $\ldots$ & 9.9 & 220333.9 & +434457.2 & -14.79 & $9.0^{\mathrm{E}}$ & 4.33 & 2.26 \\
\hline UGC 12082 & $\ldots$ & 8.8 & 223410.8 & +325137.8 & -16.13 & $10.1^{\mathrm{E}}$ & 3.95 & 2.04 \\
\hline UGC 12632 & $\ldots$ & 8.7 & 232958.7 & +405924.8 & -16.06 & $6.9^{S}$ & 4.28 & 1.88 \\
\hline UGC 12732 & $\ldots$ & 8.7 & 234039.9 & +261411.1 & -16.29 & $13.2^{\mathrm{S}}$ & 5.29 & 2.90 \\
\hline UGC 12754 & NGC 7741 & 6 & 234354.4 & +260432.2 & -18.25 & $8.9^{\mathrm{E}}$ & 4.70 & 1.51 \\
\hline
\end{tabular}

Notes. (1) Galaxy name from the UGC catalogue; (2) other common names; (3) morphological type following the classification by de Vaucouleurs (1979); (4) and (5) equatorial coordinates of the optical centre (NED); (6) absolute $B$-magnitudes (HyperLeda); (7) distance $D$ (H: deduced from the systemic velocity taken from HyperLeda corrected for Virgocentric infall and assuming $H_{0}=75 \mathrm{~km} \mathrm{~s}^{-1} \mathrm{Mpc}^{-1}$; S: Swaters et al. 2002; N: Noordermeer et al. 2005; E: Epinat et al. 2008); (8) apparent radius (HyperLeda); (9) outer H I radius as defined in this paper.

\subsection{Tilted-ring analysis}

The calculation of the kinematic lopsidedness requires the knowledge of the kinematic parameters of each galaxy. Therefore, a tilted-ring analysis of the velocity fields created from the peak value of the $\mathrm{H}_{\mathrm{I}}$ line profiles was performed (GIPSY task rotcur). The following steps were carried out: initial estimates for the centre coordinates $x_{0}$ and $y_{0}$, the inclination $i$, and the position angle PA were obtained by fitting ellipses to the H I intensity distribution out to $3 \sigma$ (GIPSY task ellfit). The systemic velocity $v_{\text {sys }}$ was taken from NED. These values were then fed into rotcur and iteratively processed, always for a combination of the receding and approaching sides (see, e.g., van Eymeren et al. 2009). The width of the rings was chosen to be half the spatial resolution, i.e., 15". We note that all pixels within a ring have equal weights. The expansion velocity $v_{\exp }$ was fixed to zero. We fixed all parameters except for the systemic velocity, which was allowed to vary with radius. After running rotcur, a mean value of $v_{\text {sys }}$ was calculated and fed into rotcur as a now best-fit and fixed value. In this way, we calculated best-fit values of $v_{\text {sys }}, x_{0}$ and $y_{0}, i$, and PA (in this given order). In a final run, we used the best-fit values for all parameters, which were fixed for all radii, and derived the rotation velocities for a combination of the receding and approaching sides.

As the velocity fields of many galaxies are quite asymmetric, we also derived rotation curves separately for both sides. The systemic velocity $v_{\text {sys }}$ and the centre coordinates $x_{0}$ and $y_{0}$ were fixed to the values derived in the first run for a combination of receding and approaching sides. The inclination and position angle were calculated for each side separately and iteratively, as described above.

We finally checked how well the derived parameters describe the observed velocity fields. We created model velocity fields by using the parameters from the final rotcur run. The model was subsequently subtracted from the original velocity field. The residuals are typically of the order of a few $\mathrm{km} \mathrm{s}^{-1}$, which means that the derived parameters describe the kinematics of the observed galaxies quite well. However, some of the nearly face-on galaxies display very disturbed velocities that appear to represent a warp, which cannot be described by a set of parameters that is constant with radius. We also found some almost edgeon galaxies, which show signs of warps in their outer morphology, whereas the velocity field is not affected at all. As already mentioned in Sect. 2, all these galaxies were removed from the final sample.

Warps in highly-inclined galaxies can easily be detected by looking at the change in scale-height with radius (Schwarzkopf \& Dettmar 2001; García-Ruiz et al. 2002). In galaxies with low inclination, lopsidedness would dominate the morphological shape so that we can be sure that the contribution of a potential warp is small along the line of sight unless the velocity field is globally twisted as mentioned above. It is more difficult to analyse the galaxies with intermediate inclinations. Here, some contamination from warps while measuring lopsidedness is quite likely.

The large-scale structure of warps has been classified as U-, S-, N-, and L-shaped (e.g., Reshetnikov \& Combes 1998; García-Ruiz et al. 2002). The first three still cause a symmetric distribution of the gas (or the stars), whereas the latter results in an asymmetric distribution, i.e., the kind of distribution we wish to study. This means that for L-shaped warps it is difficult to clearly separate warpiness from lopsidedness (at intermediate inclination). However, optical studies by Schwarzkopf \& Dettmar (2001) or Sánchez-Saavedra et al. (2003) and H i studies by García-Ruiz et al. (2002) show that the fraction of galaxies harbouring L-shaped warps is small compared to galaxies displaying U- or S-shaped warps or no warp at all.

\subsection{Kinematic lopsidedness}

Under the assumption that lopsidedness occurs as a disc response to a distorted halo produced by a galaxy interaction, an estimate of the lopsided perturbation potential that gives rise to the kinematic lopsidedness can be retrieved from the maximum rotation velocities of the sample galaxies (Jog 2002)

$\epsilon_{\text {kin }}=\frac{v_{\text {rec }}-v_{\text {appr }}}{2 v_{\mathrm{c}}}$

where $v_{\text {rec }}$ and $v_{\text {appr }}$ are the maximum rotation velocities, as measured from the plateau of the rotation curves of the receding and approaching sides, respectively, $v_{\mathrm{c}}$ is the maximum rotation velocity measured from the plateau of the combined rotation curve, and $\epsilon_{\mathrm{kin}}$ is the perturbation parameter that denotes the lopsided potential.

In many galaxies, the rotation curves are flat in the outer parts. However, in some galaxies the rotation curves rise 
monotonically to the outermost points. In those cases, we took the highest value of $v_{\mathrm{c}}$ to be an upper limit to $\epsilon_{\mathrm{kin}}$.

\section{Results}

\subsection{Rotation curves - an overview}

Figure A.1 shows the rotation curves of all sample galaxies. Black symbols represent the rotation velocities derived by combining receding and approaching sides, blue and red symbols represent the rotation velocities of the approaching and receding sides, respectively. Swaters et al. (1999) previously presented a type of velocity field in spiral galaxies where the receding and approaching sides are distinctly different, e.g., the velocities for one side rise with radius while those of the other decline (see their Fig. 2). This case is catalogued here as type 5. In addition to this, we found four types of rotation curves, which we discuss below. We present examples of each type in Fig. 2.

Type 1: For 13 galaxies (about 19\% of the whole sample), the receding and approaching sides of the rotation curves are in very good agreement indicating a very symmetric velocity distribution. Figure 2, upper row presents our results for the latetype spiral galaxy UGC 6446. Its velocity field (left panel) is very smooth and displays a typical spider pattern. The derived rotation velocities for the receding and approaching sides (right panel) differ only marginally.

Type 2: For 23 galaxies (about $33 \%$ of the whole sample), the receding and approaching sides have a constant offset. As an example, we show the irregular dwarf galaxy UGC 2080 (Fig. 2, second row). There are no local asymmetries in the velocity field, only a global offset in the iso-velocity contours.

Type 3: In ten galaxies (i.e., in $14 \%$ of all cases), receding and approaching sides are in good agreement at small radii, but show increasing differences at larger radii. A good example is the irregular dwarf galaxy UGC 9211 (Fig. 2, third row). Whereas the rotation velocities extracted from the approaching side form a typical rotation curve with a slow rise in the inner parts and a plateau in the outer parts, the rotation velocities extracted from the receding side start to decline significantly from a radius of $6 R_{25}$ on. The velocity field displays the motion of the gas that seems to be attached to the north-western part of the galaxy and probably causes the unexpected shape of the rotation curve of the receding side.

Type 4: We detected seven cases (which corresponds to $10 \%$ of all studied galaxies) where the receding and approaching sides agree well in the outer parts, but differ significantly in the inner parts. A good example is the barred spiral galaxy UGC 8863 (Fig. 2, fourth row). The velocity field shows a bar-like feature in the inner parts, which probably leads to these large discrepancies between receding and approaching sides.

Type 5: Last but not least, we found 17 galaxies (corresponding to $24 \%$ ) with differences between receding and approaching sides in both the inner and the outer parts. However, the curves change in that the rotation velocities of both sides "meet" and the decreasing side continues to decrease, whereas the increasing side continues to increase, which means that the sides flip. As an example, we show the rotation curves of the spiral galaxy UGC 7353 (Fig. 2, lower row). At a radius of about $0.8 R_{25}$ the rotation velocities extracted from the approaching side strongly increase, while the rotation velocities extracted from the receding side remain almost constant. The velocity field reveals two systems, an inner one that rotates more quickly than the outer one. This probably causes the change in the behaviour of the rotation velocities of both sides.

\subsection{Rotation curves - notes on individual galaxies}

We now discuss a few peculiar rotation curves in more detail. We only concentrate on galaxies not included in the sample of Swaters et al. (2009).

$U G C 1249$ shows a constant offset of $50 \mathrm{~km} \mathrm{~s}^{-1}$ between the rotation curves of the receding and approaching sides and is therefore classified as a type 2 galaxy. The velocity field reveals a close companion in the north, UGC 1256, which is at least twice as massive as UGC 1249. Both galaxies seem to interact with each other. While UGC 1249 is strongly affected by this process, UGC 1256 does not appear to be lopsided at all (therefore classified as type 1).

$U G C 1317$ is in a group with four other galaxies (UGC 1280, UGC 1286, UGC 1305, and UGC 1310). It shows a declining rotation curve in the outer parts. The receding and approaching sides are offset by about $50 \mathrm{~km} \mathrm{~s}^{-1}$ at all radii, which classifies this galaxy as type 2 .

UGC 1501 is very symmetric in the inner, but shows distortions in the outer parts (type 3 ). The velocity field reveals gas that seems to be attached to the western edge. Therefore, gas accretion might be the cause of the lopsidedness.

UGC 2855 has a close companion, UGC 2866, which is three times less massive. UGC 2855 shows a tail in the north-western part and additional gas attached to the southern edge. The velocity profiles of the receding and approaching sides are in good agreement in the inner parts. However, in the outer parts, the approaching rotation curve shows a decline, whereas the receding one slowly increases (type 3 ). The difference in velocity is more than $50 \mathrm{~km} \mathrm{~s}^{-1}$.

$U G C 4173$ rotates very slowly with only $36 \mathrm{~km} \mathrm{~s}^{-1}$ (there is only one more galaxy, UGC 2034, in our sample that has an even smaller rotation velocity of about $24 \mathrm{~km} \mathrm{~s}^{-1}$ ). The velocities of the receding and approaching sides differ by about $10 \mathrm{~km} \mathrm{~s}^{-1}$ across the whole radial range (type 2 ). The most remarkable thing about this galaxy is the extent to which we detect the neutral gas, which is out to almost $10 R_{25}$. Other examples are UGC 9211 (about $9 R_{25}$ ), UGC 11707 (about $7 R_{25}$ ), and UGC 11852 (about $6 R_{25}$ ). The H I disc is typically twice as extended as the optical disc in a normal spiral galaxy (Briggs et al. 1980). Irregular galaxies can have a more extended $\mathrm{H}_{\mathrm{I}}$ distribution (e.g., Huchtmeier et al. 1981). However, only one other case of an Hi disc, as extended as UGC 4173, has been published so far, which is NGC 3714 (Gentile et al. 2007).

$U G C 4458$ also appears to have a close companion that is quite mass-poor (about six times less massive than UGC 4458). The offset between the receding and approaching sides is visible at all radii (type 2) and very large reaching between 100 and $150 \mathrm{~km} \mathrm{~s}^{-1}$. The rotation velocities are unusually high with values of about $550 \mathrm{~km} \mathrm{~s}^{-1}$ (approaching side) and $350 \mathrm{~km} \mathrm{~s}^{-1}$ (receding side) at $0.75 R_{25}$, decreasing at larger radii to 400 and $300 \mathrm{~km} \mathrm{~s}^{-1}$, respectively. However, we note that the inclination is very low. From the velocity field, UGC 4458 appears to interact with its small companion.

UGC 5251 is classified as type 5. In both the inner and outer parts, the receding and approaching sides are offset by about $50 \mathrm{~km} \mathrm{~s}^{-1}$ with a change in the sides occurring at a radius of $1 R_{25}$. The velocity field is highly asymmetric.

$U G C 5532$ also shows unusually high rotation velocities with a constant offset of about $50 \mathrm{~km} \mathrm{~s}^{-1}$ between receding and approaching sides (type 2). No close companion could be detected in the Hi image. This galaxy has also been observed by the 
A\&A 530, A29 (2011)
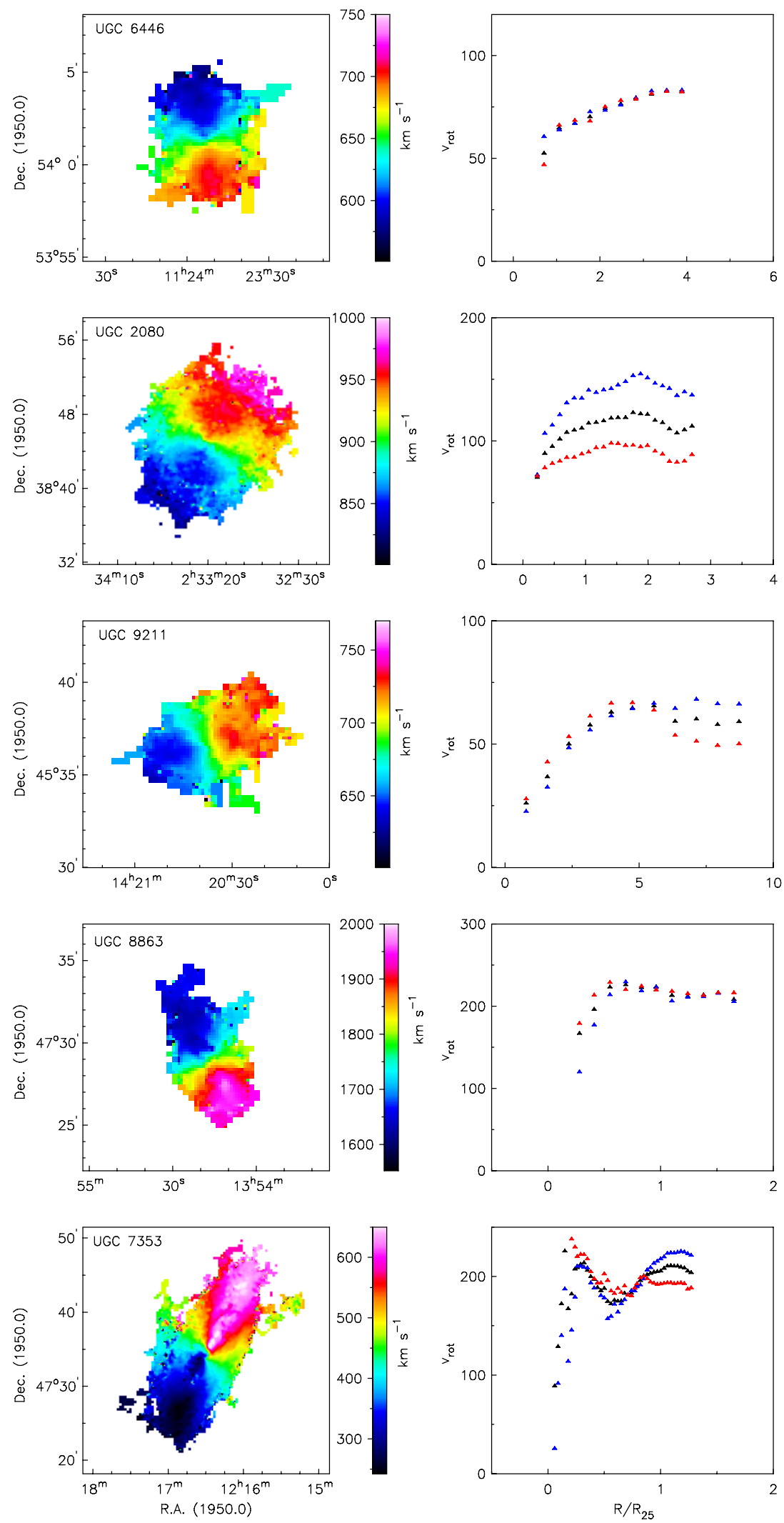

Fig. 2. Velocity fields and rotation curves (receding side only: red or light grey; approaching side only: blue or dark grey; a combination of both sides: black) of some example galaxies representing five different types of velocity patterns. From top to bottom: UGC 6446 (type 1: receding and approaching sides agree at all radii), UGC 2080 (type 2: receding and approaching sides have a constant offset), UGC 9211 (type 3: receding and approaching sides agree well at small radii, but differ significantly at large radii), UGC 8863 (type 4: receding and approaching sides differ at small radii, but agree well at large radii), UGC 7353 (type 5: the curves of receding and approaching sides change sides). 
J. van Eymeren et al.: Lopsidedness in WHISP galaxies.I.

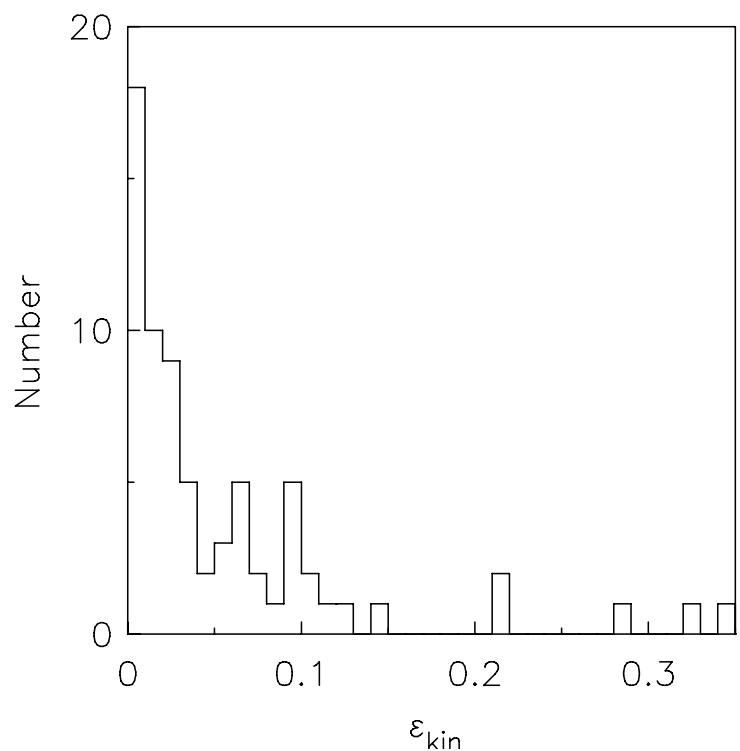

Fig. 3. The distribution of $\epsilon_{\text {kin }}$ as calculated from Eq. (1).

$G H A S P^{3}$ team, who derived a similarly looking rotation curve from the $\mathrm{H} \alpha$ velocity field (Epinat et al. 2008).

$U G C 7256$ is an extreme case of type 5 as the rotation velocities of receding and approaching sides change sides several times. The velocity field of UGC 7256 looks slightly warped. We also detected a nearby small $\mathrm{H}$ i cloud.

$U G C 7353$ is also classified as type 5 because its receding and approaching sides change sides at a radius of about $0.8 R_{25}$. The offset in the outer part is about $50 \mathrm{~km} \mathrm{~s}^{-1}$, varying in the inner parts, but is generally smaller. H I gas was detected at the eastern edge, and a very small companion is located to the northwest.

$U G C 10470$ shows a second rise in the rotation curve at $2.5 R_{25}$. The rotation curves of the receding and approaching sides are offset by about $40 \mathrm{~km} \mathrm{~s}^{-1}$ at all radii (type 2).

The combined rotation curve of UGC 12082 lies above the rotation curve for the receding and approaching sides separately. This is due to the very low inclination.

UGC 12754 has a declining rotation curve at large radii. The rotation velocities of the receding and approaching sides differ in the inner parts and are almost comparable in the outer parts (type 4).

\subsection{Kinematic lopsidedness}

As we demonstrated in the previous section, the rotation curves of galaxies allow us to examine the kinematic lopsidedness. The rotation curves of most galaxies end in a plateau such that it is possible to derive a perturbation parameter in the lopsided potential that can be measured from the kinematic data, $\epsilon_{\mathrm{kin}}$, from the maximum rotation velocities (see Sect. 3.2). As already mentioned in Sect. 3.2, $\epsilon_{\text {kin }}$ is only an estimate because the maximum rotation velocities cannot always be clearly determined. In Table 2, last column, we list $\epsilon_{\text {kin }}$ for all sample galaxies.

Figure 3 shows the distribution of $\epsilon_{\text {kin }}$. Although the mean was calculated to be about 0.056 , it becomes obvious that most galaxies have values far below the mean, which means that the kinematic lopsidedness is generally quite low. More than $60 \%$

\footnotetext{
${ }^{3}$ Gassendi HAlpha survey of SPirals.
}

of all sample galaxies have values of the perturbation parameter for the potential $\epsilon_{\mathrm{kin}}$ that are below 0.056 .

\section{Discussion}

We have analysed the rotation curves of 70 spiral and irregular galaxies. In many cases, the rotation curves extracted from the receding and approaching sides each differ remarkably. Galaxies of type 2 (such as UGC 1249 or UGC 1317), where the receding and approaching sides are offset at all radii, either have a more massive companion or are part of a group of close companions. Type 3 galaxies, i.e., galaxies that are kinematically symmetric in the inner parts, but asymmetric in the outer parts (just by comparing the rotation velocities of the receding and approaching sides), often show close companions, which are typically less massive or small $\mathrm{H}$ I clouds. Close companions are also seen for type 5 galaxies. In this class, the curves of the receding and approaching sides display a range of behaviours and therefore cross each other and change sides. This indicates that the detailed kinematic asymmetry including the spatial extent of lopsidedness strongly depends on the environment of galaxies. Type 2 and type 5 galaxies display lopsidedness at all radii. This is expected when the disc is affected by a halo that has in turn been distorted by a tidal encounter (Jog 1997). As these two types of galaxies represent $57 \%$ of the whole sample, we suggest that tidal encounters play an important role in the lopsidedness of disc galaxies. The detection of companions and H i clouds in the near vicinity of the galaxies studied here supports this scenario. In addition, gas accretion as well as ram pressure from the intergalactic medium could also cause lopsidedness, as, e.g., simulated by Mapelli et al. (2008). However, by studying NGC 891 these authors find that in this particular galaxy tidal interaction seems to be the dominant process generating lopsidedness.

Our data confirm the results by Huchtmeier (1975) of an offset between the receding and approaching sides larger than $20 \mathrm{~km} \mathrm{~s}^{-1}$. Our sample galaxies often reveal offsets as large as $50 \mathrm{~km} \mathrm{~s}^{-1}$.

We now check how well $\epsilon_{\text {kin }}$ represents the observed asymmetries in the velocity fields. We therefore concentrate on our example galaxies displayed in Fig. 2 and compare the differences in the rotation curves of the receding and approaching halves with the perturbation parameter in the lopsided potential. In UGC 6446, the rotation velocities of the receding and approaching sides are in very good agreement at all radii. Unsurprisingly, the value of $\epsilon_{\mathrm{kin}}$ is close to zero. In UGC 9211, $\epsilon_{\mathrm{kin}}$ is clearly below the mean. However, the parameter does not account for the large discrepancies in the very outer parts of the galaxy. For UGC 2080, $\epsilon_{\text {kin }}$ is very high, which reflects the constant, large difference between the receding and approaching sides. The inner parts of UGC 8863 show large differences in the rotation velocities of the receding and approaching sides. The perturbation parameter in the potential, however, is far below the mean because the velocities were averaged over the plateau where the differences are small. UGC 7353 does not have a clearly defined plateau. The maximum rotation velocities were taken from the first maximum of the rotation curves where receding and approaching sides differ only slightly. Therefore, the perturbation parameter in the potential is small, although large distortions are visible in both the very inner and very outer parts of the galaxy.

Taking into account the results for all sample galaxies, we conclude that $\epsilon_{\text {kin }}$ is a very reliable parameter if a galaxy is either globally distorted or not distorted at all. As the maximum rotation velocities are taken from a limited radial range (where the rotation curves reach their maximum), local distortions are either 
Table 2. Kinematic parameters derived from the tilted-ring analysis.

\begin{tabular}{|c|c|c|c|c|c|c|c|c|c|c|}
\hline UGC & $\begin{array}{c}v_{\text {sys }} \\
{\left[\mathrm{km} \mathrm{s}^{-1}\right]} \\
(2)\end{array}$ & $\begin{array}{c}\alpha(\mathrm{J} 2000.0) \\
{[\mathrm{h} \mathrm{m} \mathrm{s}]} \\
(3)\end{array}$ & $\begin{array}{c}\delta(\mathrm{J} 2000.0) \\
{\left[{ }^{\circ}{ }^{\prime \prime}{ }^{\prime \prime}\right]} \\
(4)\end{array}$ & $\begin{array}{c}i \\
{\left[^{\circ}\right]} \\
(5) \\
\end{array}$ & $\begin{array}{l}\mathrm{PA} \\
{\left[^{\circ}\right]} \\
(6) \\
\end{array}$ & $\begin{array}{c}v_{\mathrm{c}} \\
{\left[\mathrm{km} \mathrm{s}^{-1}\right]} \\
(7)\end{array}$ & $\begin{array}{c}v_{\mathrm{rec}} \\
{\left[\mathrm{km} \mathrm{s}^{-1}\right]} \\
(8)\end{array}$ & $\begin{array}{c}v_{\text {appr }} \\
{\left[\mathrm{km} \mathrm{s}^{-1}\right]} \\
(9)\end{array}$ & Type & (11) \\
\hline 625 & 2608 & 010055.6 & +474050.8 & 70.27 & 331.74 & 168.52 & 159.48 & 181.41 & 2 & 0.065 \\
\hline 731 & 642 & 011040.4 & +493603.8 & 59.30 & 258.16 & 72.86 & 73.45 & 73.38 & 1 & 0.001 \\
\hline 1249 & 343 & 014733.7 & +272050.3 & 51.43 & 147.57 & 65.49 & 89.23 & 46.54 & 2 & 0.326 \\
\hline 1256 & 430 & 014751.3 & +272557.1 & 71.59 & 69.44 & 114.35 & 113.92 & 115.01 & 1 & 0.005 \\
\hline 1281 & 156 & 014931.9 & +323527.3 & 73.34 & 216.86 & 59.45 & 59.74 & 60.82 & 1 & 0.009 \\
\hline 1317 & 3101 & 015114.3 & +222144.2 & 71.95 & 107.13 & 229.96 & 226.82 & 230.67 & 2 & 0.008 \\
\hline 1501 & 196 & 020116.8 & +285005.3 & 73.88 & 0.83 & 53.39 & 49.03 & 58.69 & 3 & 0.091 \\
\hline 1913 & 556 & 022715.0 & +33 3455.6 & 53.65 & 286.63 & 114.79 & 117.44 & 114.49 & 4 & 0.013 \\
\hline 2034 & 577 & 023343.0 & +403145.4 & 29.85 & 358.67 & 24.44 & 13.90 & 30.94 & 2 & 0.349 \\
\hline 2080 & 908 & 023627.2 & +3858 15.4 & 27.64 & 335.35 & 117.52 & 145.57 & 94.26 & 2 & 0.218 \\
\hline 2455 & 372 & 025941.8 & +251429.1 & 52.11 & 271.88 & 51.57 & 47.57 & 58.60 & 2 & 0.107 \\
\hline 2800 & 1177 & 034008.0 & +712417.9 & 61.87 & 285.49 & 113.26 & 111.75 & 114.19 & 3 & 0.011 \\
\hline 2855 & 1195 & 034824.8 & +700819.6 & 61.49 & 111.19 & 205.32 & 207.06 & 215.02 & 3 & 0.019 \\
\hline 2953 & 873 & 040746.8 & +694851.2 & 52.18 & 100.83 & 291.07 & 289.47 & 305.54 & 5 & 0.028 \\
\hline 3273 & 617 & 051744.9 & +533303.6 & 74.04 & 40.59 & 93.86 & 95.01 & 93.25 & 3 & 0.009 \\
\hline 3371 & 814 & 055636.4 & +751900.4 & 45.52 & 130.19 & 84.50 & 78.11 & 82.86 & 2 & 0.028 \\
\hline 3574 & 1441 & 065310.7 & +571035.5 & 31.20 & 99.79 & 125.98 & 108.89 & 123.71 & 3 & 0.059 \\
\hline 3580 & 1194 & 065530.7 & +693340.1 & 65.68 & 1.87 & 116.09 & 112.35 & 119.34 & 1 & 0.030 \\
\hline 3734 & 972 & 071228.5 & +471003.9 & 21.61 & 135.22 & 172.19 & 177.11 & 133.61 & 2 & 0.126 \\
\hline 3851 & 104 & 072854.6 & +691247.4 & 67.59 & 38.76 & 51.28 & 54.62 & 49.26 & 5 & 0.052 \\
\hline 4173 & 859 & 080705.6 & +800743.7 & 69.39 & 135.05 & 35.61 & 28.24 & 38.90 & 2 & 0.150 \\
\hline 4278 & 556 & 081359.0 & +454431.0 & 64.17 & 352.32 & 87.50 & 88.50 & 87.35 & 1 & 0.007 \\
\hline 4284 & 557 & 081440.5 & +490341.0 & 61.67 & 169.77 & 104.06 & 103.21 & 104.17 & 5 & 0.005 \\
\hline 4458 & 4753 & 083211.3 & +223334.0 & 22.10 & 289.29 & 292.78 & 400.03 & 272.95 & 2 & 0.217 \\
\hline 4543 & 1965 & 084320.3 & +454359.5 & 61.58 & 316.35 & 53.31 & 68.72 & 38.01 & 2 & 0.288 \\
\hline 4838 & 2624 & 091216.8 & +4457 17.5 & 29.14 & 304.42 & 150.33 & 129.71 & 131.79 & 2 & 0.007 \\
\hline 5079 & 551 & 093210.3 & +213010.4 & 64.01 & 202.69 & 195.31 & 193.13 & 198.56 & 4 & 0.014 \\
\hline 5251 & 1497 & 094834.3 & +332515.0 & 64.50 & 253.99 & 142.61 & 156.03 & 127.52 & 5 & 0.100 \\
\hline 5253 & 1324 & 095021.4 & +721637.8 & 36.32 & & 249.47 & 277.60 & 227.78 & 2 & 0.100 \\
\hline 5532 & 2826 & 101655.7 & +732357.7 & 35.19 & 142.79 & 300.51 & 284.11 & 260.47 & 2 & 0.039 \\
\hline 5685 & 1334 & 102919.1 & +292915.5 & 72.67 & 45.21 & 205.25 & 189.77 & 227.31 & 5 & 0.091 \\
\hline 5717 & 1687 & 103235.5 & +650256.7 & 52.30 & 10.76 & 131.30 & 132.40 & 127.69 & 5 & 0.018 \\
\hline 5721 & 537 & 103217.1 & +274008.6 & 65.53 & 279.45 & 80.29 & 76.31 & 86.31 & 2 & 0.062 \\
\hline 5789 & 738 & 103909.6 & +414112.9 & 62.49 & 35.56 & 105.14 & 104.51 & 107.04 & 1 & 0.012 \\
\hline 5829 & 628 & 104243.0 & +342726.0 & 36.94 & 191.50 & 53.79 & 48.46 & 47.94 & 5 & 0.005 \\
\hline 5918 & 335 & 104936.0 & +653158.3 & 54.04 & 238.60 & 38.07 & 36.22 & 43.82 & 3 & 0.100 \\
\hline 5997 & 1266 & 105355.4 & +734125.9 & 66.43 & 71.60 & 149.75 & 153.97 & 146.11 & 1 & 0.026 \\
\hline 6225 & 702 & 111131.1 & +554020.0 & 75.87 & 256.19 & 157.44 & 160.81 & 155.47 & 4 & 0.017 \\
\hline 6446 & 646 & 112640.7 & +534451.0 & 50.49 & 191.11 & 81.35 & 82.53 & 81.58 & 1 & 0.006 \\
\hline 6537 & 860 & 113320.8 & +470150.8 & 49.47 & 195.65 & 158.52 & 147.23 & 155.79 & 5 & 0.027 \\
\hline 6787 & 1182 & 114915.5 & +560501.6 & 68.40 & 113.51 & 237.54 & 247.13 & 228.86 & 2 & 0.039 \\
\hline 6937 & 1056 & 115735.7 & +532224.5 & 57.18 & 248.17 & 268.79 & 269.50 & 271.25 & 1 & 0.003 \\
\hline 7081 & 748 & 120528.2 & +503233.4 & 65.55 & 228.38 & 179.40 & 166.86 & 187.94 & 2 & 0.059 \\
\hline 7090 & 557 & 120600.6 & +472828.3 & 67.38 & 16.54 & 156.93 & 139.02 & 176.52 & 5 & 0.120 \\
\hline 7095 & 1081 & 120608.3 & +493504.0 & 70.52 & 345.65 & 187.04 & 196.50 & 181.48 & 2 & 0.040 \\
\hline 7151 & 266 & 120958.7 & +462724.4 & 74.89 & 281.33 & 83.55 & 85.22 & 79.92 & 1 & 0.032 \\
\hline 7256 & 1098 & 121505.5 & +331130.3 & 50.50 & 204.18 & 144.96 & 155.53 & 133.76 & 5 & 0.075 \\
\hline 7321 & 409 & 121733.7 & +223225.5 & 74.90 & 260.55 & 106.34 & 106.73 & 104.34 & 3 & 0.011 \\
\hline 7323 & 509 & 121729.4 & +453650.5 & 52.06 & 36.02 & 71.95 & 72.64 & 74.05 & 4 & 0.010 \\
\hline 7353 & 460 & 121856.8 & +471830.1 & 69.57 & 331.25 & 200.53 & 209.37 & 192.24 & 5 & 0.043 \\
\hline 7524 & 317 & 122550.4 & +333235.0 & 46.87 & 324.87 & 76.03 & 73.90 & 74.19 & 5 & 0.002 \\
\hline 7603 & 650 & 122843.5 & +225009.1 & 73.12 & 199.93 & 65.07 & 68.97 & 60.59 & 2 & 0.064 \\
\hline 7766 & 805 & 123558.5 & +2757 16.9 & 67.31 & 323.31 & 121.95 & 114.51 & 131.16 & 5 & 0.068 \\
\hline 7989 & 1190 & 125025.8 & +252956.2 & 44.19 & 32.45 & 256.47 & 243.12 & 242.60 & 4 & 0.001 \\
\hline 8863 & 1787 & 135616.3 & +471407.3 & 54.06 & 211.35 & 213.60 & 211.17 & 215.06 & 4 & 0.009 \\
\hline 9133 & 3841 & 141606.9 & +352021.6 & 51.94 & 30.36 & 237.97 & 231.80 & 245.23 & 3 & 0.028 \\
\hline 9211 & 688 & 142232.0 & +452305.0 & 46.22 & 291.13 & 61.60 & 63.72 & 60.02 & 3 & 0.030 \\
\hline 9649 & 446 & 145747.3 & +714054.7 & 56.56 & 228.21 & 88.27 & 89.76 & 89.34 & 1 & 0.002 \\
\hline
\end{tabular}


Table 2. continued.

\begin{tabular}{|c|c|c|c|c|c|c|c|c|c|c|}
\hline UGC & $\begin{array}{c}v_{\text {sys }} \\
{\left[\mathrm{km} \mathrm{s}^{-1}\right]} \\
(2)\end{array}$ & $\begin{array}{c}\alpha(\mathrm{J} 2000.0) \\
{[\mathrm{h} \mathrm{m} \mathrm{s}]} \\
(3)\end{array}$ & $\begin{array}{c}\delta(\mathrm{J} 2000.0) \\
{\left[{ }^{\circ}{ }^{\prime}{ }^{\prime \prime}\right]} \\
(4)\end{array}$ & $\begin{array}{c}i \\
{\left[{ }^{\circ}\right]} \\
(5)\end{array}$ & $\begin{array}{l}\mathrm{PA} \\
{\left[{ }^{\circ}\right]} \\
(6)\end{array}$ & $\begin{array}{c}v_{\mathrm{c}} \\
{\left[\mathrm{km} \mathrm{s}^{-1}\right]} \\
(7)\end{array}$ & $\begin{array}{c}v_{\mathrm{rec}} \\
{\left[\mathrm{km} \mathrm{s}^{-1}\right]} \\
(8)\end{array}$ & $\begin{array}{c}v_{\text {appr }} \\
{\left[\mathrm{km} \mathrm{s}^{-1}\right]} \\
(9)\end{array}$ & Type & (11) \\
\hline 9858 & 2628 & 152641.5 & +403353.7 & 69.98 & 75.80 & 170.73 & 184.91 & 154.54 & 5 & 0.089 \\
\hline 10359 & 908 & 162057.0 & +652332.9 & 43.82 & 278.01 & 127.97 & 122.73 & 126.05 & 1 & 0.013 \\
\hline 10470 & 1359 & 163239.8 & +78 1207.9 & 36.78 & 300.11 & 130.96 & 142.00 & 117.28 & 2 & 0.094 \\
\hline 11670 & 777 & 210333.4 & +295349.9 & 67.34 & 335.63 & 173.00 & 177.36 & 168.55 & 2 & 0.026 \\
\hline 11707 & 901 & 211430.8 & +264404.1 & 65.37 & 55.72 & 97.71 & 89.37 & 102.82 & 5 & 0.069 \\
\hline 11852 & 5837 & 215559.6 & +275357.0 & 46.03 & 192.35 & 181.95 & 163.18 & 171.37 & 2 & 0.023 \\
\hline 11861 & 1481 & 215622.3 & +731540.6 & 48.30 & 216.95 & 152.18 & 149.95 & 148.84 & 1 & 0.004 \\
\hline 11891 & 457 & 220331.4 & +434511.1 & 46.26 & 115.49 & 87.50 & 81.05 & 84.74 & 5 & 0.021 \\
\hline 12082 & 805 & 223411.3 & +325124.3 & 20.56 & 145.25 & 87.19 & 58.33 & 45.02 & 2 & 0.076 \\
\hline 12632 & 426 & 233000.6 & +405941.5 & 49.49 & 38.38 & 65.93 & 69.07 & 69.00 & 5 & 0.001 \\
\hline 12732 & 747 & 234040.1 & +261400.0 & 40.27 & 14.33 & 79.34 & 83.81 & 78.05 & 3 & 0.036 \\
\hline 12754 & 753 & 234354.1 & +260438.5 & 49.46 & 344.85 & 116.74 & 116.93 & 120.25 & 4 & 0.014 \\
\hline
\end{tabular}

Notes. (1) The UGC number; (2) to (6) the kinematic parameters as iteratively calculated from the tilted-ring analysis for a combination of receding and approaching sides: (2) the systemic velocity, (3) and (4) equatorial coordinates of the dynamic centre, (5) the inclination; (6) the position angle which is measured in anti-clockwise direction from the north to the receding half of the galaxy; (7) to (9) the maximum rotation velocities as measured from the plateaus of the rotation curves: $v_{\mathrm{c}}$ (for a combination of receding and approaching sides), $v_{\text {rec }}$ (as measured from the receding side), and $v_{\text {appr }}$ (as measured from the approaching side); (10) the type of rotation curve as defined in Sect. $4.1 ;(11)$ the perturbation parameter in the lopsided potential as measured from the kinematic data (see Eq. (1)).

not included or overestimated, depending on whether we have a type 4 or type 3 galaxy. In about $20 \%$ of all sample galaxies, the H I content does not extend much further than $R_{25}$, so that the maximum rotation velocities were averaged over a radial range within $R_{25}$. In all other galaxies, the maximum rotation velocities were measured at radii beyond $R_{25}$.

To summarise, the most reliable way to quantify the kinematic lopsidedness is to compare the rotation curves of the receding and approaching sides.

As mentioned in the introduction, the main assumption about deriving rotation curves is that they are azimuthally symmetric, which results in a symmetric mass distribution. However, as our analysis shows, there can be huge differences between the rotation curves extracted from the receding and approaching sides. Since the occurrence of asymmetries in disc galaxies is quite high, extra care has to be taken when analysing the rotation curves and the mass distribution in those objects.

\section{Summary}

We have obtained the rotation curves of 70 spiral and irregular galaxies selected from the WHISP survey by performing a tiltedring analysis of the $\mathrm{H}_{\mathrm{I}}$ data. We considered the receding and approaching sides separately and discussed the differences in the velocities. The galaxies can be divided into five different types in terms of the behaviour of the rotation curves of the receding and approaching sides, and have symmetric velocity fields, global distortions or local distortions at different radii. The most common types are type 2 where the velocities of the receding and approaching sides show a constant offset over all radii, often as high as $50 \mathrm{~km} \mathrm{~s}^{-1}$, and type 5 where the curves of receding and approaching sides change sides. Both types of galaxies are either members of a group of close companions, or have either a close companion of higher or lower mass or an $\mathrm{H}$ i cloud nearby. We suggest that these global distortions are the result of the disc response to a halo that was distorted by a tidal encounter.
Furthermore, we have calculated the perturbation parameter for the lopsided potential from the kinematic asymmetry, $\epsilon_{\mathrm{kin}}$, which gave us a mean value of about 0.056 (averaged over all sample galaxies). However, this parameter either under- or overestimates lopsidedness if there are local distortions.

To quantify lopsidedness at all radii and help determine a better idea of its physical origin(s), a more detailed study of lopsidedness has to be carried out. Morphological lopsidedness can easily be measured on all scales. A detailed study of the morphological lopsidedness will allow us to shed some light on the mechanisms that lead to lopsidedness in general. This analysis will be done in Paper II.

Acknowledgements. The authors would like to thank the anonymous referee for the constructive feedback which helped to improve this paper.

We wish to thank Thijs van der Hulst for providing us with the WHISP data cubes before they became publicly available. C.J. would like to thank DFG (Germany) and INSA (India) for supporting a visit to Germany in October 2007 under INSA-DFG Exchange Programme, during which this collaboration was started. We made extensive use of NASA's Astrophysics Data System (ADS) Bibliographic Services and the NASA/IPAC Extragalactic Database (NED) which is operated by the Jet Propulsion Laboratory, California Institute of Technology, under contract with the National Aeronautics and Space Administration. We acknowledge the usage of the HyperLeda database (http: //leda.univ-lyon $1 . f r)$.

\section{References}

Angiras, R. A., Jog, C. J., Omar, A., \& Dwarakanath, K. S. 2006, MNRAS, 369, 1849

Angiras, R. A., Jog, C. J., Dwarakanath, K. S., \& Verheijen, M. A. W. 2007, MNRAS, 378, 276

Baldwin, J. E., Lynden-Bell, D., \& Sancisi, R. 1980, MNRAS, 193, 313

Binney, J., \& Tremaine, S. 1987, Galactic dynamics, ed. J. Binney, \& S. Tremaine

Briggs, F. H., Wolfe, A. M., Krumm, N., \& Salpeter, E. E. 1980, ApJ, 238, 510 de Vaucouleurs, G. 1979, ApJ, 227, 380

Epinat, B., Amram, P., Marcelin, M., et al. 2008, MNRAS, 388, 500

García-Ruiz, I., Sancisi, R., \& Kuijken, K. 2002, A\&A, 394, 769

Gentile, G., Salucci, P., Klein, U., \& Granato, G. L. 2007, MNRAS, 375, 199

Huchtmeier, W. K. 1975, A\&A, 45, 259

Huchtmeier, W. K., Seiradakis, J. H., \& Materne, J. 1981, A\&A, 102, 134 
Jog, C. J. 1997, ApJ, 488, 642

Jog, C. J. 2002, A\&A, 391, 471

Jog, C. J., \& Combes, F. 2009, Phys. Rep., 471, 75

Kannappan, S. J., \& Fabricant, D. G. 2001, in Galaxy Disks and Disk Galaxies, ed. J. G. Funes, \& E. M. Corsini, ASP Conf. Ser., 230, 449

Mapelli, M., Moore, B., \& Bland-Hawthorn, J. 2008, MNRAS, 388, 697

Noordermeer, E., van der Hulst, J. M., Sancisi, R., Swaters, R. A., \& van Albada,

T. S. 2005, A\&A, 442, 137

Reshetnikov, V., \& Combes, F. 1998, A\&A, 337, 9

Rhee, G., Valenzuela, O., Klypin, A., Holtzman, J., \& Moorthy, B. 2004, ApJ, 617,1059

Richter, O., \& Sancisi, R. 1994, A\&A, 290, L9

Rix, H., \& Zaritsky, D. 1995, ApJ, 447, 82

Sánchez-Saavedra, M. L., Battaner, E., Guijarro, A., López-Corredoira, M., \& Castro-Rodríguez, N. 2003, A\&A, 399, 457
Schwarzkopf, U., \& Dettmar, R. 2001, A\&A, 373, 402

Shane, W. W., \& Bieger-Smith, G. P. 1966, Bull. Astron. Inst. Netherlands, 18, 263

Swaters, R. A., Schoenmakers, R. H. M., Sancisi, R., \& van Albada, T. S. 1999, MNRAS, 304, 330

Swaters, R. A., van Albada, T. S., van der Hulst, J. M., \& Sancisi, R. 2002, A\&A 390,829

Swaters, R. A., Sancisi, R., van Albada, T. S., \& van der Hulst, J. M. 2009, A\&A, 493,871

van der Hulst, J. M., Terlouw, J. P., Begeman, K. G., Zwitser, W., \& Roelfsema, P. R. 1992, in Astronomical Data Analysis Software and Systems I, ed. D. M. Worrall, C. Biemesderfer, \& J. Barnes, ASP Conf. Ser., 25, 131

van Eymeren, J., Marcelin, M., Koribalski, B. S., et al. 2009, A\&A, 505, 105

van Eymeren, J., Jütte, E., Jog, C., Stein, Y., \& Dettmar, R.-J. 2011, A\&A, 530, A30

Pages 11 to 14 are available in the electronic edition of the journal at http://www . aanda.org 
J. van Eymeren et al.: Lopsidedness in WHISP galaxies.I.

\section{Appendix A: Rotation curves of all sample galaxies}

Here we present the rotation curves of all sample galaxies

(Fig. A.1). Red and blue symbols mark the rotation velocities of the receding and approaching sides, respectively. The rotation curve of a combination of the receding and approaching sides is shown in black. 

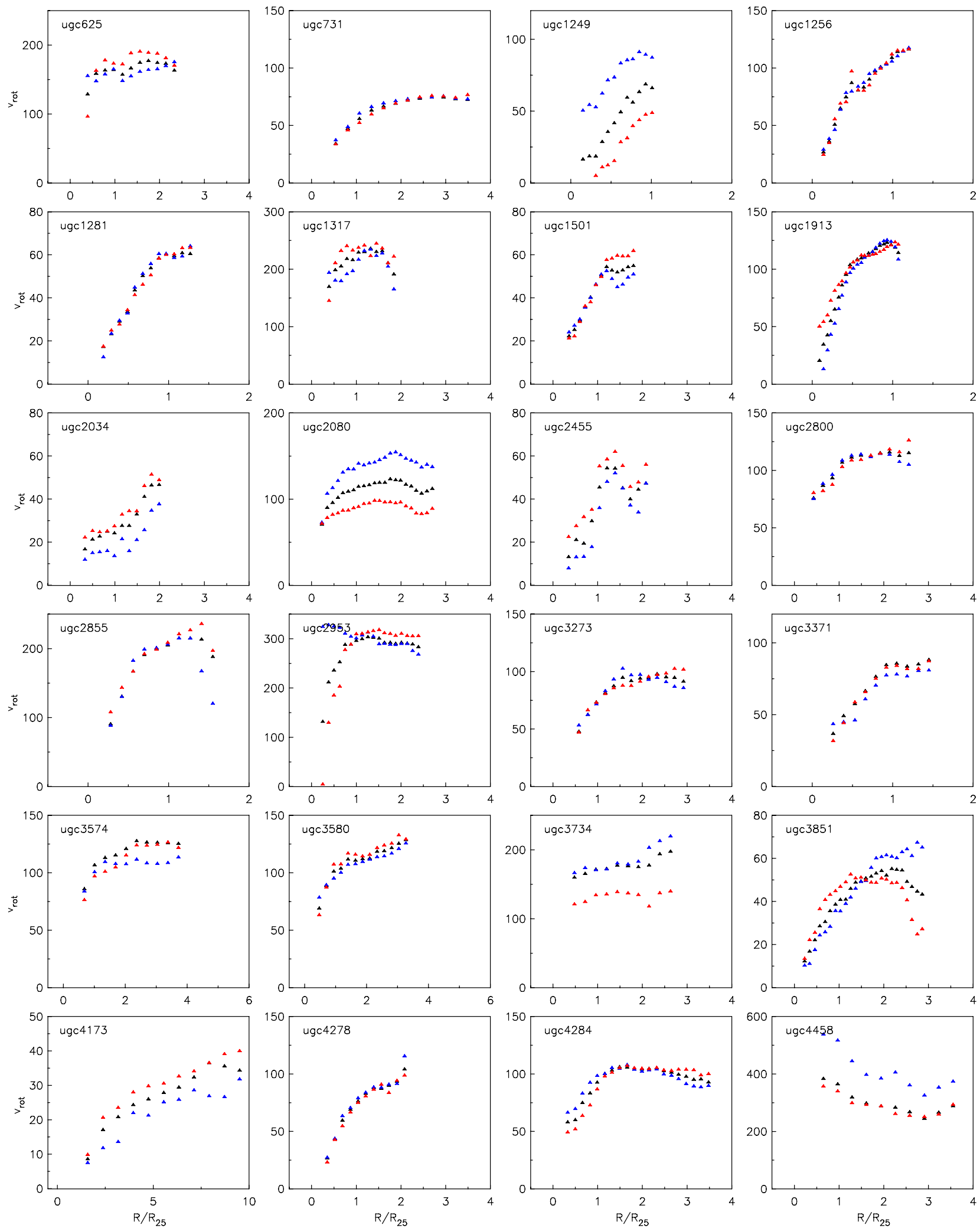

Fig. A.1. The rotation curves of all sample galaxies extracted from the receding (red, light grey), approaching (blue, dark grey), and a combination of both sides (black). 
J. van Eymeren et al.: Lopsidedness in WHISP galaxies.I.
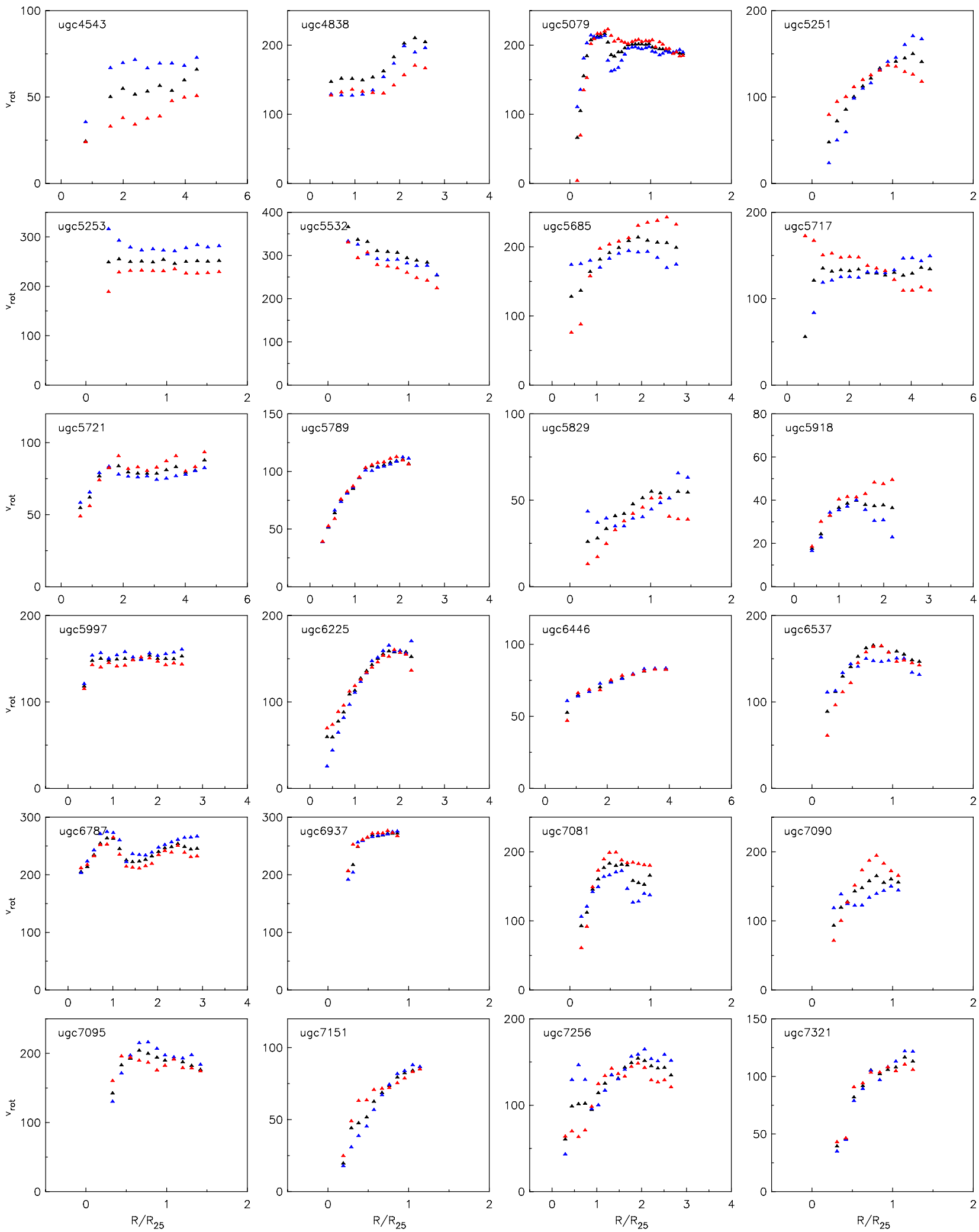

Fig. A.1. continued. 

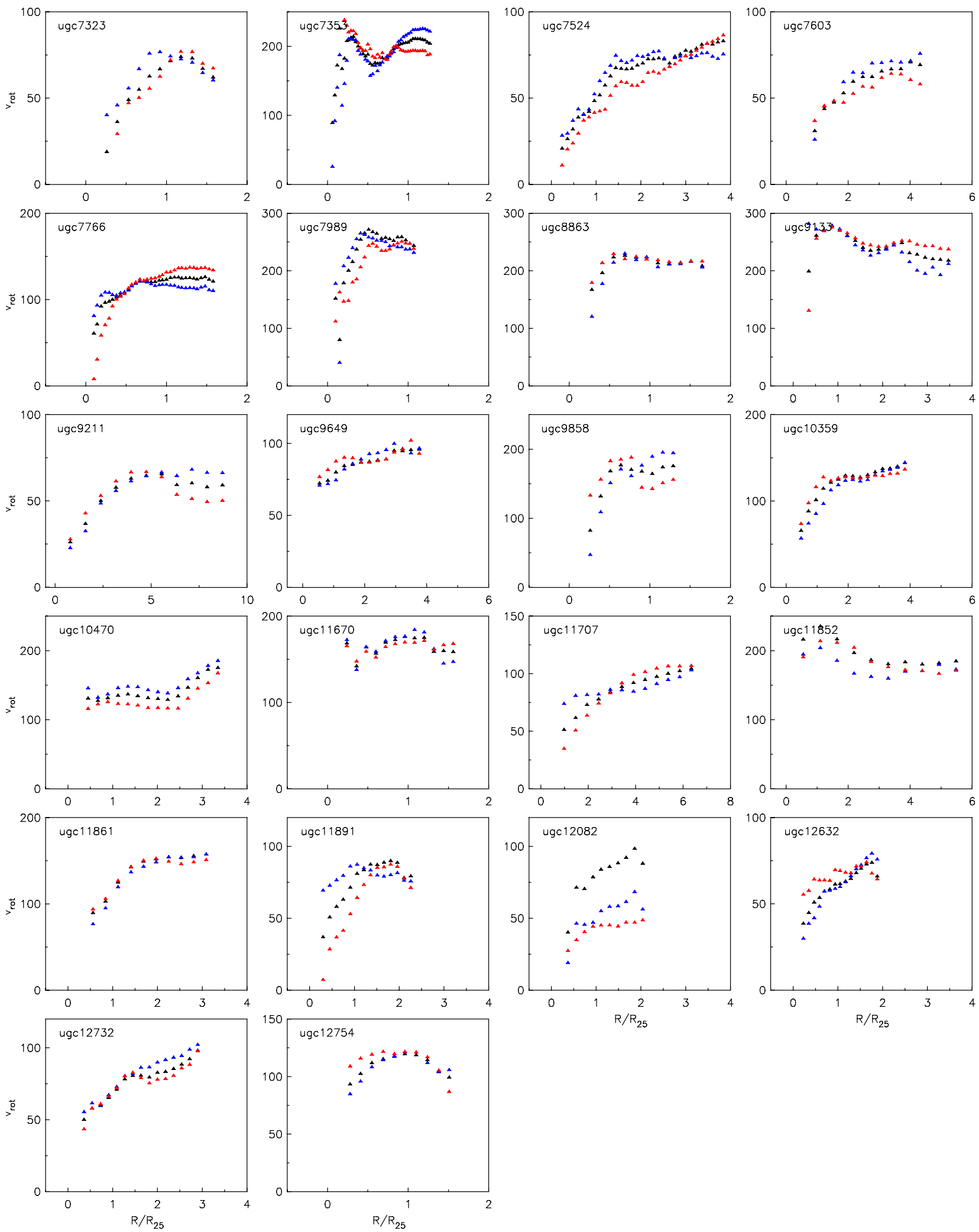

Fig. A.1. continued. 